\title{
Pole orientation and triaxial ellipsoid shape of (25143) 1998 SF36, a target asteroid of the MUSES-C* mission
}

\author{
Youhei Ohba ${ }^{1}$, Masanao Abe $^{1}$, Sunao Hasegawa ${ }^{1}$, Masateru Ishiguro ${ }^{1}$, Tomasz Kwiatkowski ${ }^{2}$, \\ Francois Colas $^{3}$, Budi Dermawan ${ }^{4}$, and Akira Fujiwara ${ }^{1}$ \\ ${ }^{1}$ Planetary Science Division, The Institute of Space and Astronautical Science (ISAS), 3-1-1 Yoshinodai, Sagamihara, \\ Kanagawa 229-8510, Japan \\ ${ }^{2}$ Poznan Astronomical Observatory, ul. Sloneczna 36, PL-60-286 Poznan, A. Mickiewicz University, Poland \\ ${ }^{3}$ Institut de Mécanique Céleste, 77 Av. Denfert Rochereau, 75014 Paris, France \\ ${ }^{4}$ School of Science, The University of Tokyo, Bunkyo, Tokyo 113-0033, Japan
}

(Received March 14, 2003; Revised July 1, 2003; Accepted July 10, 2003)

\begin{abstract}
The near-earth asteroid (25143) 1998 SF36 is a target body for the Japanese sample-return MUSES-C mission. We present here its pole orientation and triaxial ellipsoid shape, using light curve data obtained with three telescopes at Kiso, Mitaka, and Pic du Midi. The solution obtained for the pole orientation has ecliptic latitude $\lambda=320 \pm 30^{\circ}$ and ecliptic longitude $\beta=-75 \pm 12^{\circ}$. The estimated triaxial ellipsoid shape is $a / b=2.1$ and $b / c=1.7$ assuming $m=0.03$, which is the coefficient of the empirical relation between the light curve amplitude and the phase angle for S-type asteroids (Zappalà et al., 1990). We also found $m$ to be related to asteroid surface roughness, using a light curve simulator.
\end{abstract}

Key words: Asteroid, shape, pole orientation, surface roughness, mission target.

\section{Introduction}

The near-earth asteroid (25143) $1998 \mathrm{SF} 36$ ( $a=1.33 \mathrm{AU}$, $\left.e=0.28, i=1.72^{\circ}\right)$ is a target body for the Japanese sample-return MUSES-C mission (Fujiwara et al., 2002; Farquhar et al., 2002). This asteroid was discovered on September 26, 1998 by the Lincoln Near-Earth Asteroid Research program and made a close approach to the Earth at the end of March, 2001. The taxonomic type of 1998 SF36 has been determined from spectroscopic observations between wavelengths of 0.5 and $2.5 \mu \mathrm{m}$, placing it in the S(IV) group (Binzel et al., 2001). Radar observations have suggested two preliminary pole solutions (ecliptic longitude $\lambda$, ecliptic latitude $\beta)$ : either $\left(320^{\circ} \pm 30^{\circ},-75^{\circ} \pm 15^{\circ}\right)$ or $\left(230^{\circ} \pm 15^{\circ}\right.$, $\left.-5^{\circ} \pm 15^{\circ}\right)$. They have also allowed determination of a first approximation of the asteroid's shape, characterizing it as an ellipsoid $630 \pm 60 \mathrm{~m}$ in length and $250 \pm 30 \mathrm{~m}$ in width (Ostro et al., 2001). Many observers have obtained light curve data for this asteroid, using optical photometric observations. Dermawan et al. (2002) found that its synodic rotational period is $12.13 \pm 0.02$ hours. In this paper, we describe its pole orientation and triaxial ellipsoid shape, determined using light curve data obtained with three telescopes at Kiso, Mitaka, and Pic du Midi.

*The spacecraft MUSES-C had been launched successfully on May 9 2003 , and the spacecraft was renamed "HAYABUSA" which means "falcon" in Japanese.

Copy right (c) The Society of Geomagnetism and Earth, Planetary and Space Sciences (SGEPSS); The Seismological Society of Japan; The Volcanological Society of Japan; The Geodetic Society of Japan; The Japanese Society for Planetary Sciences.

\section{Observations and Data Reduction}

We carried out optical photometric observations of 1998 SF36 during its 2001 apparition, using the 105-cm Kiso Schmidt telescope with the SITe 2 KCCD, the 50-cm NAOMitaka telescope with the Astromed CCD (Dermawan et al., 2002) and the 105-cm Pic du Midi telescope with the Thomson CCD (Michałowski et al., 2000). The observational conditions are summarized in Table 1 . We used exposure times ranging from $60 \mathrm{sec}$. to $360 \mathrm{sec}$. during the entire run.

The raw image data were bias-subtracted and normalized using the flat-field images. We used the software $I R A F$ for aperture photometry, with the exception of the Pic du Midi frames, which were reduced with the STARLINK package. The photometry of this asteroid was determined relative to a number of comparison stars - five stars were used in every frame. The apparent magnitudes of the comparison stars were determined by observing several distinct standard stars on the same night. The magnitudes of our standard stars were derived from the Landolt catalogue (Landolt, 1992).

\section{Pole Orientation and Triaxial Ellipsoid Shape 3.1 Epoch method}

To estimate the orientation of the pole position, we used the Epoch method described by Magnusson (1986). The light curve minima were selected as the "Standard Feature" $(S F S)$, because we detected the epochs of only the light curve minima in Kiso data. Assuming this is the true pole orientation, the following equation holds:

$$
\frac{T_{i}-T_{0}}{P}-n_{i}=\frac{\theta_{i}-\theta_{0}}{2 \pi},
$$

where $T_{0}$ is the time at the first $S F, T_{i}$ is the time at the $i$-th $S F . P$ is the sidereal rotational period, and $n_{i}$ denotes 
Table 1. Observational condition.

\begin{tabular}{|c|c|c|c|c|c|c|c|c|}
\hline Date & Mag & $r(\mathrm{AU})$ & $\Delta(\mathrm{AU})$ & S-T-O $\left(^{\circ}\right)$ & EcLon $\left({ }^{\circ}\right)$ & EcLat $\left(^{\circ}\right)$ & Tel & Filter \\
\hline $2001 / 2 / 20$ & 16.4 & 1.15 & 0.17 & 22 & 175 & 11 & $\mathrm{P}$ & $\mathrm{R}$ \\
\hline $2001 / 2 / 21$ & 16.3 & 1.14 & 0.17 & 22 & 175 & 12 & $\mathrm{P}$ & $\mathrm{R}$ \\
\hline $2001 / 2 / 22$ & 16.2 & 1.14 & 0.16 & 21 & 175 & 12 & $\mathrm{P}$ & $\mathrm{R}$ \\
\hline $2001 / 2 / 25$ & 16.0 & 1.13 & 0.15 & 21 & 177 & 13 & $\mathrm{P}$ & $\mathrm{R}$ \\
\hline $2001 / 2 / 26$ & 15.9 & 1.12 & 0.14 & 21 & 177 & 14 & $\mathrm{P}$ & $\mathrm{R}$ \\
\hline $2001 / 3 / 13$ & 14.6 & 1.06 & 0.08 & 26 & 189 & 23 & M & I \\
\hline $2001 / 3 / 15$ & 14.5 & 1.06 & 0.07 & 28 & 192 & 26 & M & I \\
\hline $2001 / 3 / 26$ & 14.0 & 1.02 & 0.04 & 57 & 232 & 42 & $\mathrm{~K}$ & BVRI \\
\hline $2001 / 3 / 29$ & 14.2 & 1.01 & 0.04 & 71 & 255 & 44 & $\mathrm{~K}$ & $\mathrm{R}$ \\
\hline $2001 / 3 / 31$ & 14.6 & 1.00 & 0.04 & 81 & 271 & 42 & $\mathrm{~K}$ & BVRI \\
\hline $2001 / 4 / 1$ & 14.8 & 1.00 & 0.04 & 85 & 278 & 41 & $\mathrm{~K}$ & $\mathrm{R}$ \\
\hline $2001 / 8 / 22$ & 17.9 & 1.30 & 0.32 & 21 & 355 & -7 & K & $\mathrm{R}$ \\
\hline $2001 / 8 / 23$ & 17.9 & 1.31 & 0.32 & 20 & 355 & -7 & K & $\mathrm{R}$ \\
\hline $2001 / 8 / 24$ & 17.9 & 1.31 & 0.32 & 19 & 354 & -7 & $\mathrm{~K}$ & $\mathrm{R}$ \\
\hline $2001 / 8 / 25$ & 17.9 & 1.32 & 0.32 & 17 & 354 & -7 & K & $\mathrm{R}$ \\
\hline
\end{tabular}

Mag : Predicted apparent magnitude.

$r$ : Heliocentric distance (AU),

$\Delta$ : Geocentric distance (AU).

S-T-O: Phase (Sun-Asteroid-Observer) angle $\left(^{\circ}\right)$.

EcLon: Observed-centered ecliptic longitude of the asteroid $\left({ }^{\circ}\right)$.

EcLat: Observed-centered ecliptic latitude of the asteroid $\left({ }^{\circ}\right)$.

Tel: Telescope (P: Pic du Midi, M: Mitaka, K: Kiso).

Table 2. Time of $S F$ and the orientation of $P A B$.

\begin{tabular}{lrrrrr}
\hline Date & $2001 / 3 / 13$ & $2001 / 3 / 15$ & $2001 / 3 / 26$ & $2001 / 3 / 31$ & $2001 / 4 / 1$ \\
\hline Time $\left(T_{i}-T_{0}\right)$ (hour) & 0 & 45.58 & 315.16 & 435.97 & 459.85 \\
Ecliptic longitude $(P A B)\left({ }^{\circ}\right)$ & 1.77 & 4.36 & 32.10 & 54.69 & 58.64 \\
Ecliptic latitude $(P A B)\left({ }^{\circ}\right)$ & -12.85 & -13.99 & -22.33 & -21.26 & -20.31 \\
Apparent mean rotational period $\left(T_{i}-T_{0}\right) / n_{i}$ (hour) & - & 12.15 & 12.12 & 12.11 & 12.10 \\
\hline
\end{tabular}

Table 3. Light curve amplitude and orientation of $P A B$.

\begin{tabular}{lccc}
\hline Date & $2001 / 2 / 20,21,22,25,26$ & $2001 / 3 / 13,15$ & $2001 / 8 / 22,23,24,25$ \\
\hline Amplitude (mag.) & 0.78 & 0.73 & 0.86 \\
Ecliptic longitude $\left(P A B,^{\circ}\right)$ & 346.59 & 2.23 & 165.34 \\
Ecliptic latitude $\left(P A B,^{\circ}\right)$ & -7.02 & -13.04 & 4.38 \\
Phase angle $\left({ }^{\circ}\right)$ & 21.26 & 27.20 & 19.12 \\
\hline
\end{tabular}

the number of rotations between $T_{0}$ and $T_{i} . \theta_{0}$ and $\theta_{i}$ are the astrocentric longitude of the phase angle bisector $(P A B)$ at $T_{0}$ and $T_{i}$, respectively. If the astrocentric longitude of $P A B$ is constant, the synodic rotational period is constant and equal to the sidereal rotational period. The derived value of $S F$ and the ecliptic longitude and latitude of $P A B$ are shown in Table 2.

We define $\delta$ in the following equation:

$$
\delta=\sum_{i}^{N} \sqrt{\left[\left(\frac{T_{i}-T_{0}}{P}-n_{i}-\frac{\theta_{i}-\theta_{0}}{2 \pi}\right)^{2}\right] /(N-1) .}
$$

We calculated $\delta$ for each assumed pole orientation, and the results are shown in Fig. 1 for a sidereal rotational period of 12.16 hours. In Fig. 1, there are four solutions for the pole orientation corresponding to minima of $\delta$. The apparent mean rotational period decreased with the direction of $P A B$ changing in an anticlockwise direction as shown in Table 2 and Fig. 2. This indicates that the asteroid's rotation is retrograde - that is, the pole orientation of the asteroid is south of the ecliptic plane - and allows us to eliminate the two solutions in which the latitude of the pole orientation is positive. This conclusion does not change even if we consider the uncertainties in $T_{i}$.

\subsection{Amplitude method}

To estimate the orientation of the pole position, we used a second approach: the Amplitude method, described by 


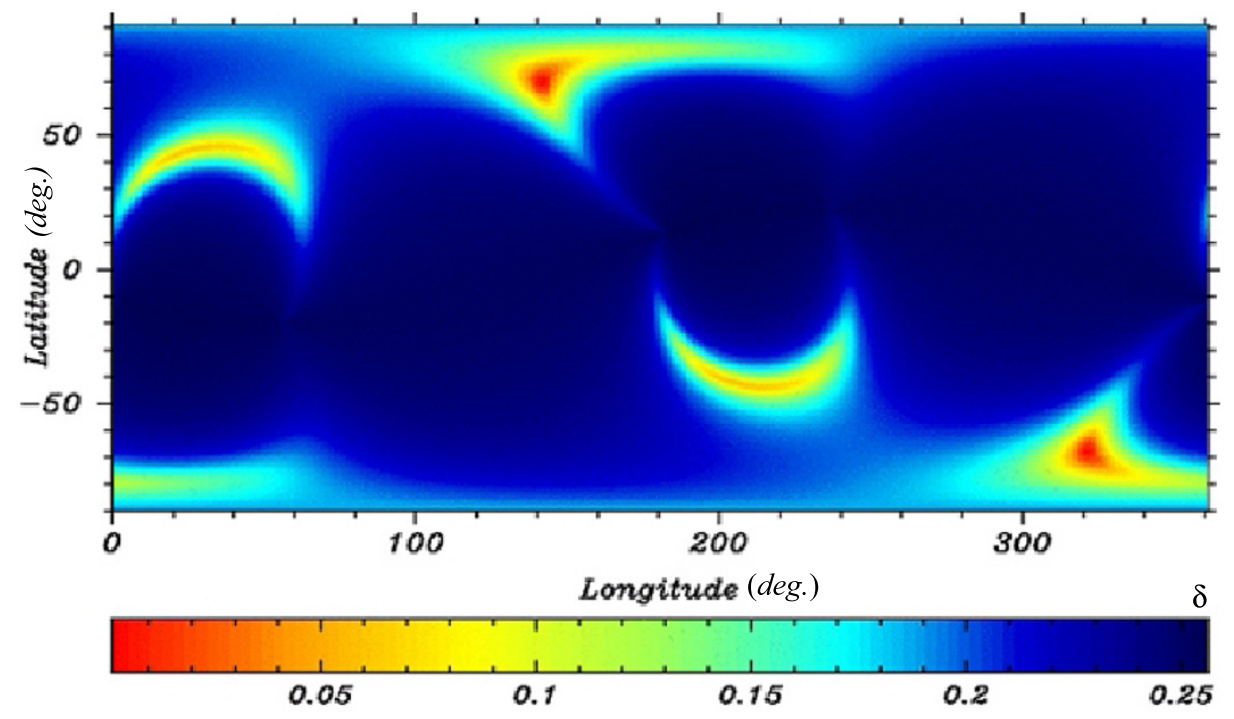

Fig. 1. Probability map of pole orientation for 1998 SF36 using the Epoch method. Lower $\delta$ regions indicate a higher probability of the pole orientation.

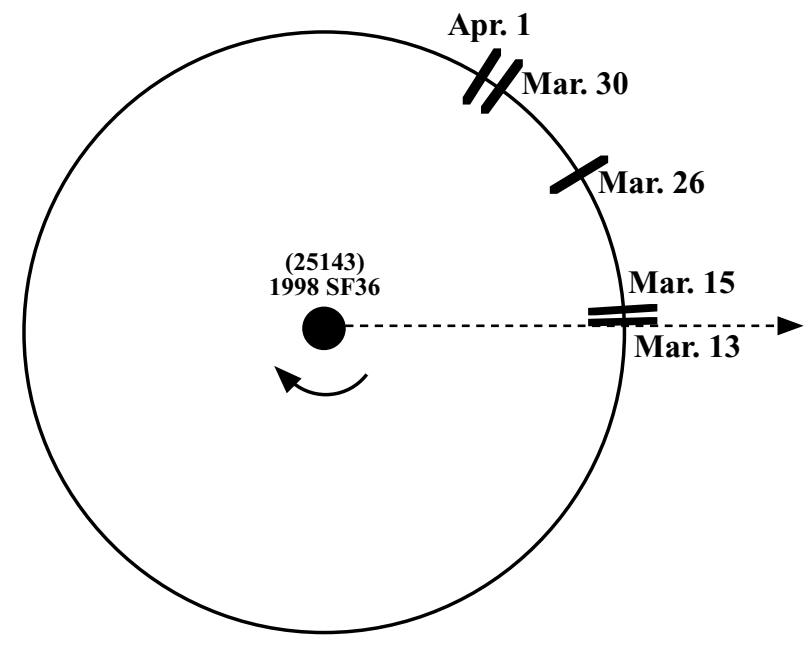

Fig. 2. The observing geometries in March and April, 2001, are shown relative to 1998 SF36 (center). The circular scale is the ecliptic longitude of the phase angle bisector $(\mathrm{PAB})$. The direction of the dashed line is that of the equinox. As the apparent mean rotational period decreased with changes in the direction of PAB in the anticlockwise direction, the rotation of 1998 SF36 is retrograde (see Table 2 and the text for details).

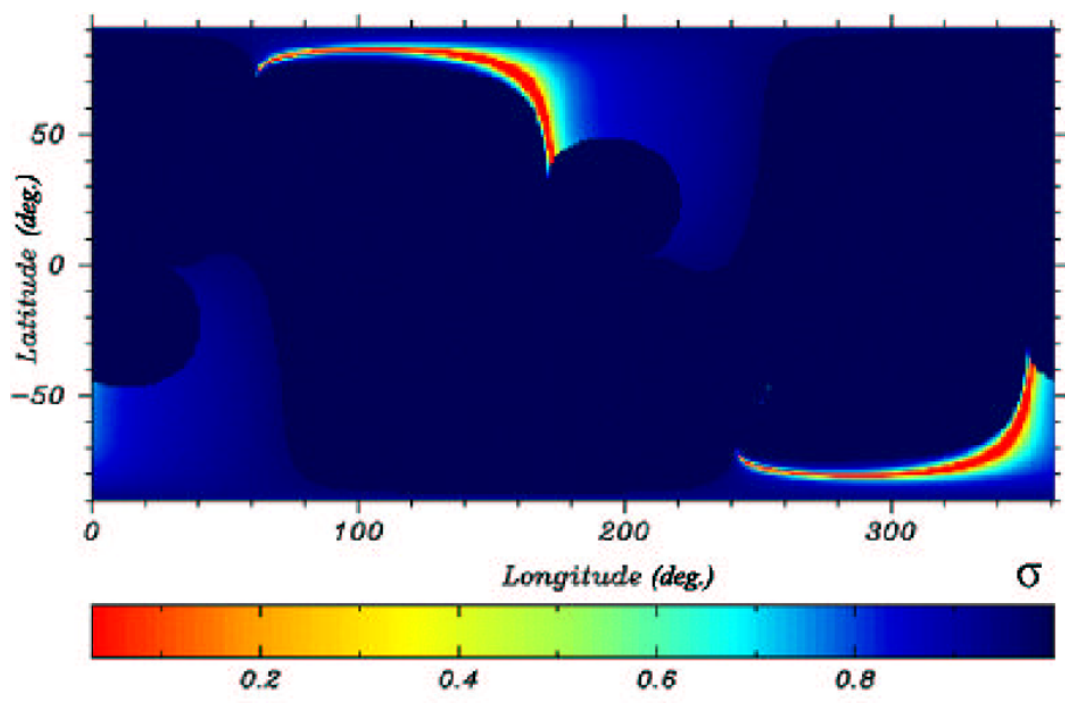

Fig. 3. Probability map of pole orientation for 1998 SF36 using the Amplitude method. Lower $\sigma$ regions indicate a higher probability of the pole orientation. 

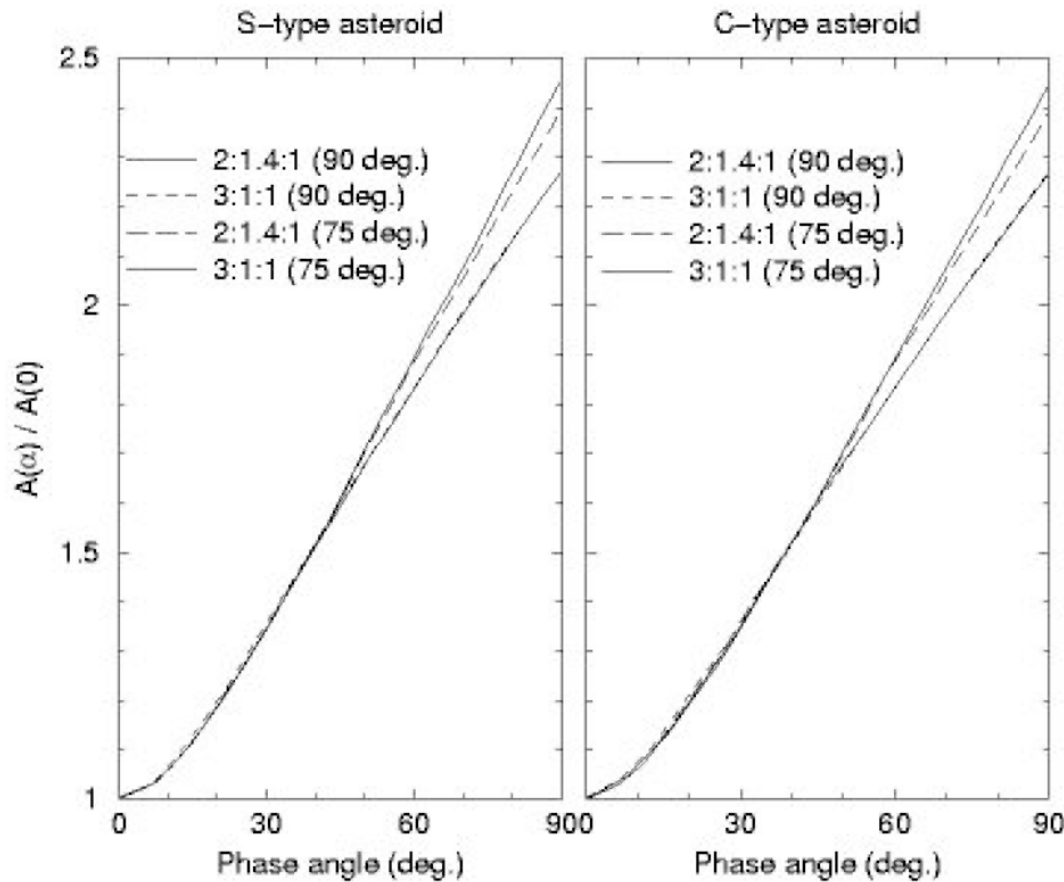

Fig. 4. The relationship between the phase angle and the amplitude curve normalized at the phase angle of $0^{\circ}$, i.e., $A(\alpha) / A\left(0^{\circ}\right)$, for S-type asteroids (left) and C-type asteroids (right), where the surface is assumed to be smooth. The Hapke parameters for each taxonomic type are from Helfenstein and Veverka (1989). The relation was deduced as a function of shape ( $a: b: c)$ and some aspect angles (given in parentheses). The slope of the curve, $m$, becomes the same, at least at phase angles below $40^{\circ}$.

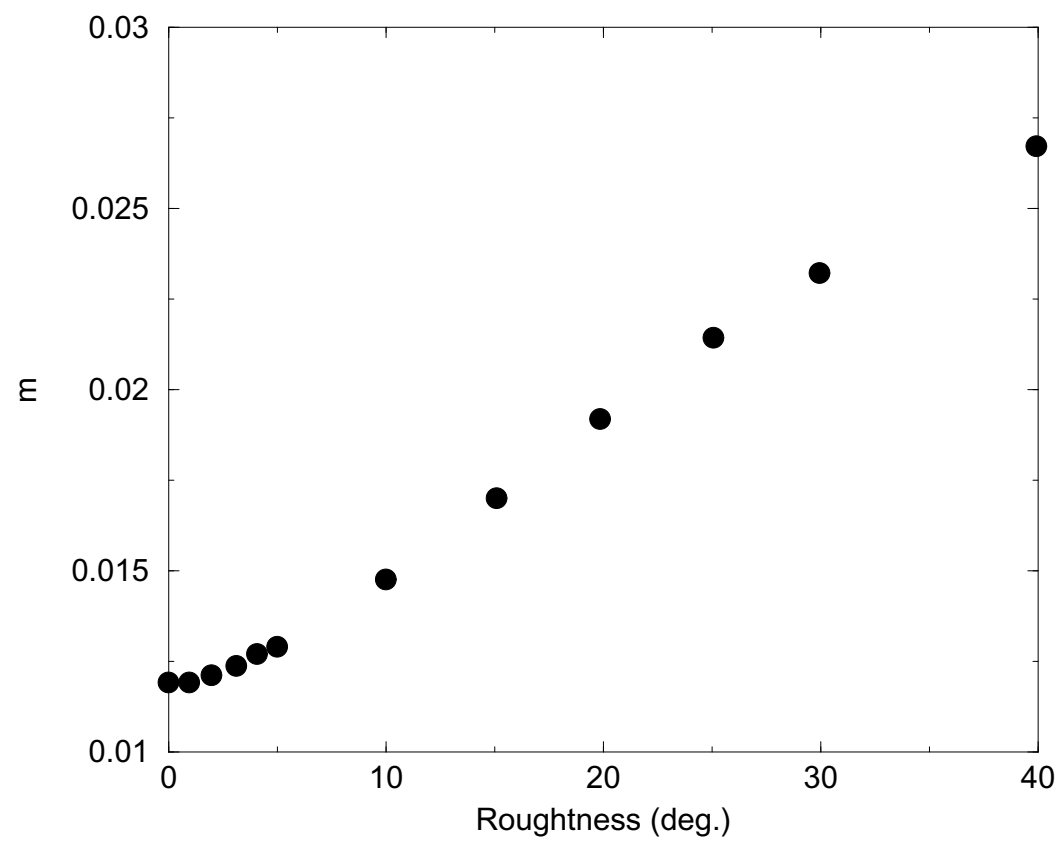

Fig. 5. The relationship between the roughness and the coefficient $m$.

Magnusson (1986). Assuming a triaxial ellipsoid shape, the light curve amplitude can be written as follows:

$A(\phi, \alpha)=1.25\left\{\log \left[\frac{(b / c)^{2} \cos ^{2}(\phi)+\sin ^{2}(\phi)}{(b / c)^{2} \cos ^{2}(\phi)+(b / a)^{2} \sin ^{2}(\phi)}\right]\right\}$

$$
\cdot(1+m \alpha)
$$

where $\phi$ is the aspect angle (the angle between pole orientation and $P A B), \alpha$ is the phase angle (the Sun-asteroid- observer angle), and $a, b$, and $c$ are the lengths of the principal axes of the ellipsoid, with the constraint $a \geq b \geq c$. We assume that the albedo is constant over the whole surface of the asteroid. $m$ is the coefficient of the empirical relation between light curve amplitude and phase angle (Zappalà et al., 1990). We adopted $m=0.03$, which is the value for S-type asteroids reported by Zappalà et al. (1990). We measured the amplitude at three epochs and the results are shown in Table 3. 
Table 4. Obtained triaxial ellipsoid shape with various surface roughness $\bar{\theta}$

\begin{tabular}{ccccccc}
\hline$m$ (roughness $\bar{\theta})$ & $0.012\left(0^{\circ}\right)$ & $0.015\left(10^{\circ}\right)$ & $0.020\left(20^{\circ}\right)$ & $0.025\left(30^{\circ}\right)$ & $0.027\left(40^{\circ}\right)$ & $0.030\left(50^{\circ}\right)$ \\
\hline$a / b$ & 2.7 & 2.6 & 2.3 & 2.1 & 2.1 & 1 \\
$b / c$ & 1.4 & 1.4 & 1.6 & 1.7 & 1.7 & 1.7 \\
\hline
\end{tabular}

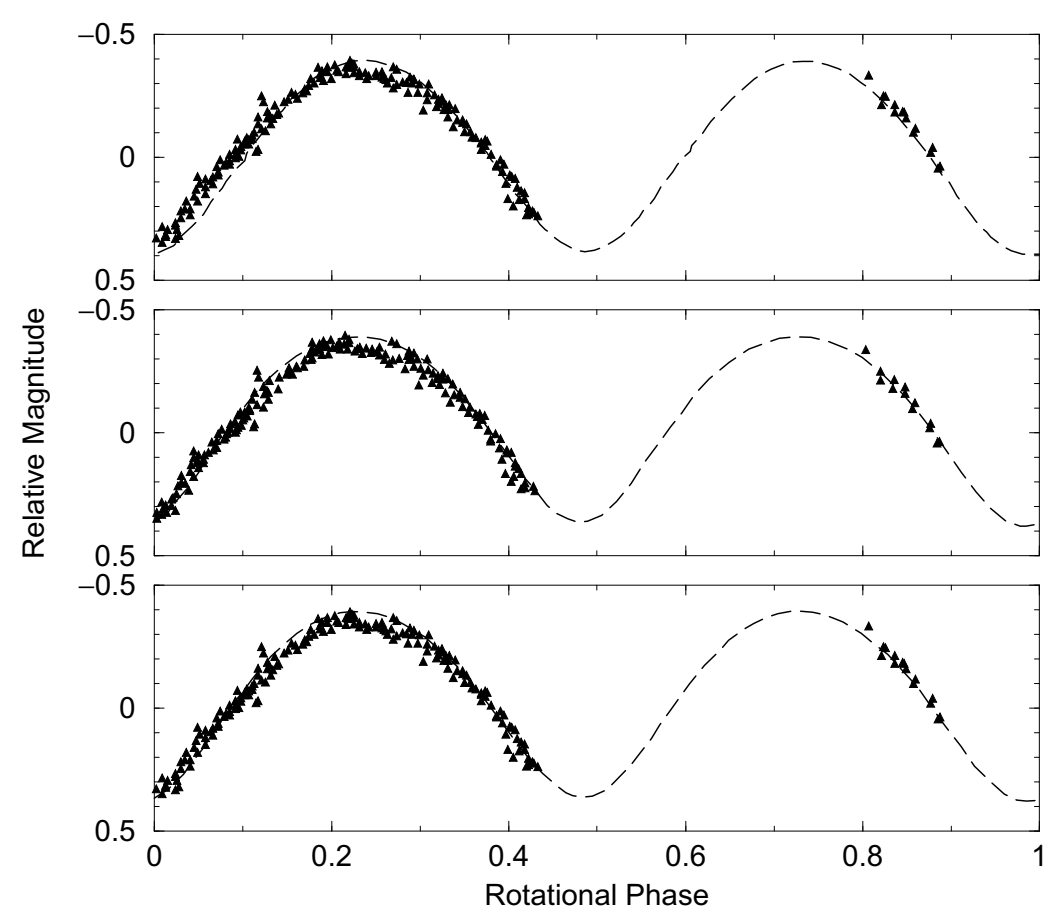

Fig. 6. Simulated light curve (dashed line) using the obtained solution, and observed light curve (symbols). The observed light curves were obtained at Mitaka on March 13 and 15, 2001. The light curves assume a rotational period of 12.15 hours, and assume $m=0.012$ (smooth surface) and $a: b: c=1: 0.37: 0.26$ (top panel), for $m=0.02$ (medium roughness with $\bar{\theta}=20^{\circ}$ ) and $a: b: c=1: 0.44: 0.27$ (middle panel), and for $m=0.03$ (heavy roughness with $\bar{\theta}=50^{\circ}$ ) and $a: b: c=1: 0.44: 0.27$ (bottom panel).

For each assumed pole orientation, we calculated the standard deviation $\sigma$ of the solution for the triaxial ellipsoid shape of $a / b$ and $b / c$ using the three datasets shown in Table 3 and Eq. (3). The distribution of $\sigma$ is shown in Fig. 3. As shown in Fig. 3, the solution using the Epoch method suggests that the pole orientation is almost perpendicular to the ecliptic plane. This can be understood from the fact that the observed amplitude is always large and has almost the same value at each epoch. The final solution is the intersection between the plausible regions of $\delta$ and $\sigma$, derived from the Epoch method and the Amplitude method, respectively. That is, the value of the solution is $\lambda=320^{\circ} \pm 30^{\circ}$, $\beta=-75^{\circ} \pm 12^{\circ}$. The resulting pole orientation yields a triaxial ellipsoid, based on Eq. (3), with $b / a=2.1_{-0.3}^{+0.5}$ and $b / c=1.7 \pm 0.1$.

\section{The Relation between $m$ and Surface Roughness $\bar{\theta}$}

Zappalà et al. (1990) suggested that the coefficient $m$ varies with taxonomic type. Karttunen and Bowell (1989), however, argued that the variation of the light curve amplitude is almost the same for S-type and C-type asteroids, using a light curve simulator and the Lumme-Bowell scattering law (Bowell and Lumme, 1979). Here, we propose that the coefficient $m$ is related to the surface roughness $\bar{\theta}$. The roughness is the "mean slope angle" defined by $\tan \bar{\theta}=(2 / \pi) \int_{0}^{\pi / 2} a\left(\theta^{\prime}\right) \tan \theta^{\prime} d \theta^{\prime}$. The angles of tilt are assumed to be distributed uniformly in azimuth and described by the function $a\left(\theta^{\prime}\right)$, where $\theta^{\prime}$ is the normal to mean surface angle. We constructed a light curve simulator (Ohba, 2002) using the Hapke scattering law (Hapke, 1993), and verified that the simulator could reproduce the light curves of (243) Ida, adopting the pole orientation and shape estimated by Dotto et al. (1995). We also confirmed the mean phase curves for C- and S-type asteroids (Bowell and Lumme, 1979) using the Hapke parameters of C- and S-type asteroids reported by Helfenstein and Veverka (1989). Using this light curve simulator, we calculated the variations of the light curve amplitude with phase angle for various shape and aspect angles. As shown in Fig. 4, when the surface roughness is the same, the variation of the light curve amplitude is almost the same (at least below phase angles of $40^{\circ}$ ). The taxonomic type, ellipsoid shape, and the aspect angle play only minor roles in the relation between the light curve amplitude and the phase angle.

We next calculated $m$, defined as follows:

$$
\frac{A(\alpha)}{A(0)}=(1+m \alpha)
$$

for various surface roughnesses (see Fig. 5) and the results 


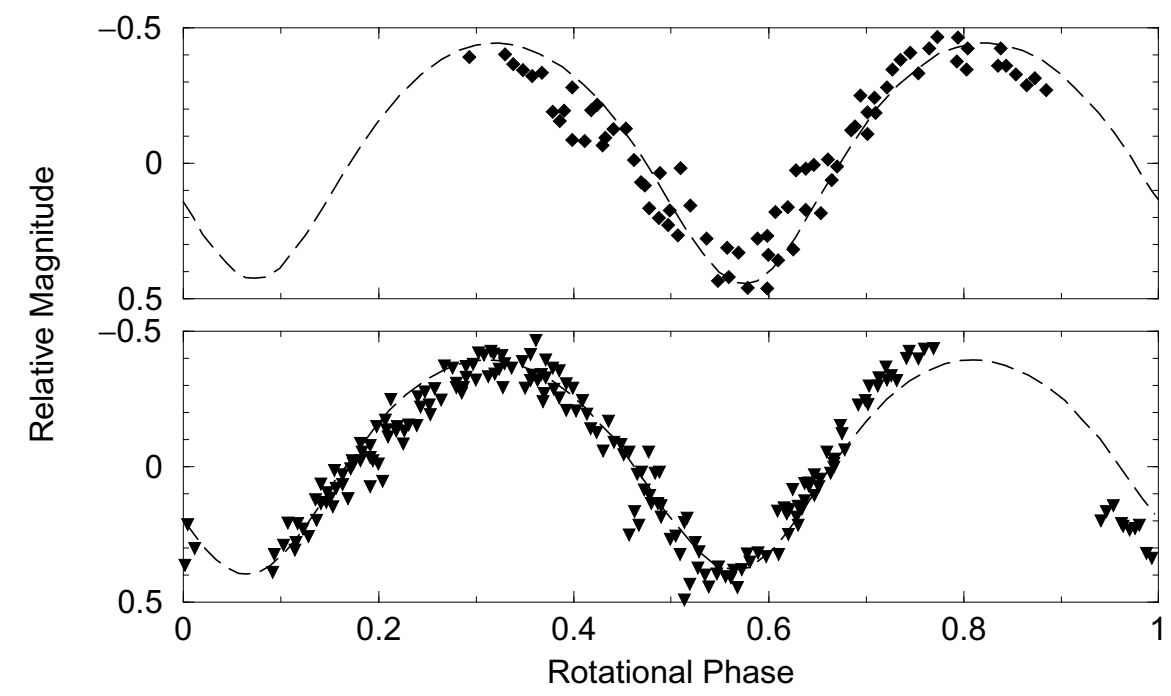

Fig. 7. Simulated light curve (dashed line) using observed light curves obtained at Kiso between August 22 and 26 (top panel) and at Pic du Midi between February 20 and 26 (bottom panel). The light curve (dashed line) is the same as that in the middle panel of Fig. 6.

are plotted in Fig. 4.

As shown in Fig. 5, it appears that the coefficient $m$ increases with surface roughness. We recalculated the pole orientation and triaxial ellipsoid shape for 1998 SF36, relating $m$ to the surface roughness through the results plotted in Fig. 5. The pole orientation was not different from the value obtained in the case of $m=0.03$, but the shape of the triaxial ellipsoid, especially $a / b$, was different if $m$ is taken to be related to surface roughness. The results are shown in Table 4.

\section{Discussion}

Zappalà $e$ t al. (1990) suggested that the coefficient $m$ differs for S-type and C-type asteroids. In this study, however, we have shown that the coefficient $m$ varies with surface roughness $\bar{\theta}$ of the Hapke scattering model. As the coefficient $m$ does not vary with other parameters of the Hapke model, as described in the previous section, this seems to indicate that the surface roughnesses of S-type and C-type asteroids are different.

Using the Epoch and Amplitude methods, we determined one solution of pole orientation and triaxial ellipsoid shape for the mission target asteroid 1998 SF36. The results obtained were essentially consistent with those of previous radar observations (Ostro et al., 2001), but we showed that the asteroid has a triaxial ellipsoid shape, while Ostro et al. (2001) assumed $b=c$. Figures 6 and 7 show the simulated light curve obtained using our solution, together with observed light curves. It is not possible to determine the surface roughness by comparing the observed and simulated light curves. However, if we could confirm the triaxial ellipsoid shape by some other means, it would be possible to determine the surface roughness. The light curve amplitude at zero phase angle is dependent on the triaxial ellipsoid shape and the aspect angle, but not the surface roughness. If we had light curve data near the zero of the phase angle, it would be possible to determine the triaxial ellipsoid shape and surface roughness independently.
The asteroid 1998 SF36 will make another close approach to the Earth in June 2004, while the spacecraft MUSES-C will arrive at the asteroid in June 2005, and continue its in situ observations there for the subsequent 5 months. We will thus have an opportunity to compare the results of the ground-based observations with the results of in situ observations of this asteroid in the near future.

Acknowledgments. We are grateful to Prof. T. Mukai and Prof. V. Zappalà for reviewing this paper. This research was partially supported by the Ministry of Education, Science, Sports and Culture (Grants-in-Aid for Scientific Research 14340135, and 13740271). TK was supported by Polish KBN Grant 2 P03D 007 18. Pic du Midi data were reduced with the CCLRC STARLINK package.

\section{References}

Binzel, R. P., A. S. Rivkin, S. J. Bus, J. M. Sunshine, and T. H. Burbine, MUSES-C Target Asteroid (25143) 1998 SF36: A Reddened Ordinary Chondrite, Meteoritics \& Planetary Science, 36(8), 1167-1172, 2001.

Bowell, E. and K. Lumme, Colorimetry and Magnitudes of Asteroids, in Asteroids, Edited by T. Gehrels, pp. 132-169, University of Arizona Press, 1979.

Dermawan, B., T. Nakamura, H. Fukushima, H. Sato, F. Yoshida, and Y. Sato, CCD Photometry of the MUSES-C Mission Target: Asteroid (25143) 1998 SF36, Publications of the Astronomical Society of Japan, 54, 635-640, 2002.

Dotto, E., M. A. Barucci, and M. Fulchignoni, Ground-based Ida model comparison with the first Galileo image, Planet. Space Sci., 43(5), 683689,1995

Farquhar, R., J. Kawaguchi, C. Russell, G. Schwehm, J. Veverka, and D. Yeomans, Space craft Exploration of Asteroids: The 2001 Perspective, in Asteroid III, Edited by W. F. Bottke, Jr., A. Cellino, P. Paolicchi, and R. P. Binzel, pp. 367-378, 2002.

Fujiwara, A., J. Kawaguchi, and K. T. Uesugi, Role of asteroid sample return mission (MUSES-C) in asteroid research, Advances in Space Research, 2002 (submitted).

Hapke, B., Theory of Reflectance and Emittance Spectroscopy, Cambridge University Press, 1993.

Helfenstein, P. and J. Veverka, Physical characterization of asteroid surfaces from photometric analysis, in Asteroids II, Edited by Richard P. Binzel, T. Gehrels, M. S. Matthews, pp. 524-556, University of Arizona Press, 1989.

Karttunen, H. and E. Bowell, Modeling asteroid brightness variations. IIThe interpretability of light curves and phase curves, Astronomy and Astrophysics, 208, 320-326, 1989.

Landolt, A. U., UBVRI photometric standard stars in the magnitude range 
11.5-16.0 around the celestial equator, Astronomical Journal, 104, 340371, 436-491, 1992.

Magnusson, P., Distribution of spin axes and senses of rotation for 20 large asteroids, Icarus, 68, 1-39, 1986.

Michałowski, T., W. Pych, J. Berthier, A. Kryszczynska, T. Kwiatkowski, J. Boussuge, S. Fauvaud, P. Denchev, and R. Baranowski, CCD photometry, spin and shape models of five asteroids: 225, 360, 416, 516, and 1223 , Astronomy and Astrophysics Supplement, 146, 471-479, 2000.

Ohba, Y., Physical Model of (25143) 1998 SF36, The Target Asteroid of Sample Return Mission, MUSES-C-Estimation from Ground Observation and Simulation-, Master's thesis, Univ. of Tokyo, 2002.
Ostro, S. J., L. A. M. Benner, M. C. Nolan, J. D. Giorgini, R. F. Jurgens, R. Rose, and D. K. Yeomans, Rader observations of Asteroid 25143 (1998 SF36), American Astronomical Society, DPS meeting, 33, 41.13, 2001. Zappalà, V., A. Cellino, A. M. Barucci, M. Fulchignoni, and D. F. Lupishko, An analysis of the amplitude-phase relationship among asteroids, Astronomy and Astrophysics, 231, 548-560, 1990.

Y. Ohba, M. Abe (e-mail: abe@planeta.sci.isas.ac.jp), S. Hasegawa, M. Ishiguro, T. Kwiatkowski, F. Colas, B. Dermawan, and A. Fujiwara 